\title{
Multisensor Processing Algorithms for Underwater Dipole Localization and Tracking Using MEMS Artificial Lateral-Line Sensors
}

\author{
Saunvit Pandya, ${ }^{1}$ Yingchen Yang, ${ }^{1}$ Douglas L. Jones, ${ }^{2}$ Jonathan Engel, ${ }^{1}$ and Chang Liu ${ }^{1}$ \\ ${ }^{1}$ Micro and Nanotechnology Laboratory, University of Illinois, Urbana-Champaign, Urbana, IL 61801, USA \\ ${ }^{2}$ Coordinated Science Laboratory, University of Illinois, Urbana-Champaign, Urbana, IL 61801, USA
}

Received 1 January 2006; Revised 12 June 2006; Accepted 16 July 2006

\begin{abstract}
An engineered artificial lateral-line system has been recently developed, consisting of a 16-element array of finely spaced MEMS hot-wire flow sensors. This represents a new class of underwater flow sensing instruments and necessitates the development of rapid, efficient, and robust signal processing algorithms. In this paper, we report on the development and implementation of a set of algorithms that assist in the localization and tracking of vibrational dipole sources underwater. Using these algorithms, accurate tracking of the trajectory of a moving dipole source has been demonstrated successfully.
\end{abstract}

Copyright (c) 2006 Saunvit Pandya et al. This is an open access article distributed under the Creative Commons Attribution License, which permits unrestricted use, distribution, and reproduction in any medium, provided the original work is properly cited.

\section{MOTIVATION}

In nature, almost all species of fish use arrays of cilium-like haircell sensors in a lateral-line configuration for flow sensing and near-field hydrodynamic imaging [1]. Each haircell sensor in the lateral line is capable of measuring local fluid flow velocity. Fish utilize the lateral-line organ for a rich set of behaviors including schooling, navigation, predator avoidance, and prey capture.

Manmade underwater vehicles currently use technologies such as sonar or optical systems for navigation and imaging. However, these established methods have limitations. Active sonar, for example, may reveal the location of the source. Furthermore, many sonar systems rely on pulse-echo width analysis. This method has limited resolution and does not work well in close range. Optical systems cannot operate in deep or murky waters.

In light of these limitations, a biomimetic flow sensing system inspired by the fish lateral line could augment or complement current technologies. Potential applications would include imaging and maneuvering control for autonomous underwater vehicles (AUVs), intrusion detection (ID) systems, and hydro-robotics. For example, underwater vehicles and platforms equipped with artificial lateral lines could detect intruders (e.g., a swimmer) based on the hydrodynamic signature, thereby allowing unprecedented methods of threat monitoring.
An engineering equivalent of the biological lateral-line organ, an artificial lateral line, has never been developed. This is primarily due to the fact that commercially available flow sensors are typically bulky and therefore not amenable for high-density array integration.

However, recent advancement in micromachining and MEMS makes it possible to mimic functions and structures of biological sensors such as lateral lines [2,3]. MEMS sensors can offer high sensitivity and high-resolution capabilities with low power consumption, small footprint, and at low cost (due to integrated-circuit-style batch production). Researchers have made MEMS sensors based on many transduction principles and for many applications, including temperature sensors, accelerometers $[4,5]$, gyroscopes, pressure sensors, tactile sensors [6-9], flow sensors [10-13], and multimodal sensors $[6,14]$. MEMS flow sensors based on principles such as hot-wire anemometry and biomimetic haircell sensing have also been developed [10, 13, 15-23].

Recently, our group invented an engineered artificial lateral-line system, consisting of a 16-element array of finely spaced hot-wire flow sensors. Fast and efficient algorithms are needed to analyze complex spatial-temporal input from the sensor array for perception of hydrodynamic activities. Here, we report on our progress with the design and implementation of algorithms complementing the artificial lateralline system for a complete biomimetic hardware-software solution. 


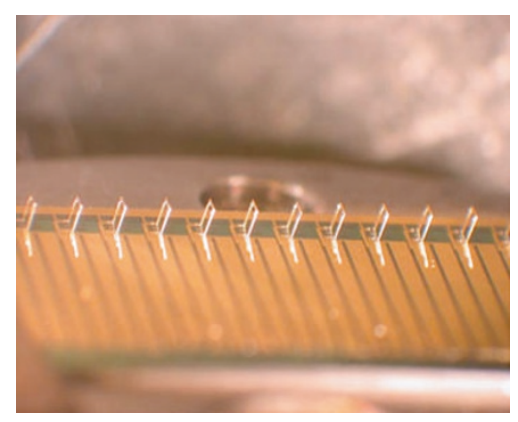

(a)

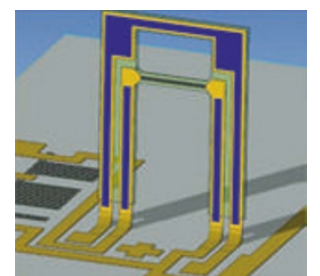

(b)

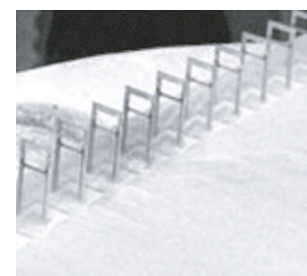

(c)
FIGURE 1: (a) An optical micrograph of an artificial lateral line, consisting of a linear array of hot-wire anemometers. (b) Schematic diagram of a single raised hot-wire sensor. (c) An SEM micrograph of the same array.

\section{SENSOR DESCRIPTION}

The artificial lateral line consists of a linear array of hot-wire anemometers (HWAs) $[12,15,16,19,20]$. In Figure 1, an array of 16 HWA sensors with $1 \mathrm{~mm}$ spacing between each is shown. An individual HWA consists of a thermal resistive element (hot wire) and operates on the principle of convective heat loss. During operation, the hot-wire element is heated above the ambient temperature using an electrical current. When it is exposed to a flow medium, the fluid convectively removes heat from the hot wire and causes its temperature to drop and its resistance value to change.

The density of the sensors approaches that of the biological lateral line in some fish. Through the use of micromachining technology such high-density arrays can be made, together with analog integrated circuits [15] for local signal conditioning.

The HWA sensor offers high performance in terms of sensitivity. The fabricated MEMS HWA can sense flow at the order of $10 \mathrm{~mm} / \mathrm{s}$. Another advantage of the MEMS HWA sensor is the desired frequency range. The micromachined hot-wire anemometer has a viable frequency range from 0 (DC) to $\sim 10 \mathrm{kHz}$, thus spanning the entire frequency range for hydrodynamic events of interest [12].

\section{FLUID THEORY OVERVIEW}

Using the lateral-line sensing organ, fish can detect water flow disturbances underwater. One of the simplest and most commonly encountered forms of disturbance is an acoustic dipole [12]. Biologists have studied fish lateral-line response

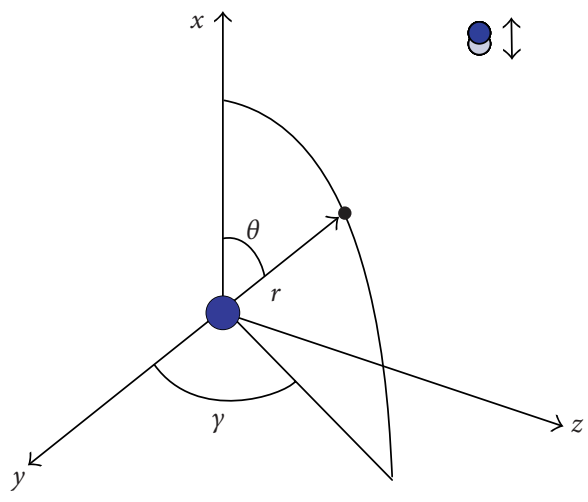

FIGURE 2: Schematic of analytical model (dipole at origin and observation point at $r, \theta, \gamma)$ [25].

to acoustic dipoles extensively and found that fish can locate the source of a dipole and track its movement [1]. Therefore, we choose to investigate the performance of our artificial lateral-line sensor in response to an oscillating dipole source.

The acoustic dipole model has been well established $[1,22,24,25]$. The pressure and velocity distributions, respectively, can be described according to an abridged version of the model as

$$
\begin{gathered}
p(r, \theta)=-\frac{\rho \omega a^{3} U_{o} \cos (\theta)}{2 r^{2}}, \\
v_{\text {flow }}(r, \theta)=\left(a^{3} U_{o} \frac{\cos (\theta)}{r^{3}}\right) \hat{e}_{r}+\left(\frac{a^{3} U_{o}}{2} \frac{\sin (\theta)}{r^{3}}\right) \hat{e}_{\theta} .
\end{gathered}
$$

Equation (1) relates the scalar pressure field of a dipole in the local flow region to the dipole diameter $a$, the density $\rho$, the observation distance $r$ and angle $\theta$, the angular frequency $\omega$, as well as the dipole's initial vibrational velocity amplitude $U_{0}$. Equation (2) describes the local fluid flow velocity (vector field) as a function of the initial velocity, position, and dipole diameter. The position of the observation point, as well as the coordinate description, is shown in Figure 2.

The root-mean-square ( $\mathrm{rms}$ ) velocity distribution in response to an oscillating dipole, as per the analytical model presented in (1)-(2), is shown in Figure 3(a). Figure 3(b) shows the experimental response of an HWA to a dipole stimulus. The experimental output of the sensor matches pertinent profile information predicted by the theoretical model. The difference between the two profiles can be attributed to the directional sensitivity of the sensor. A detailed explanation of this phenomenon is beyond the scope of this paper.

\section{EXPERIMENTAL SETUP}

Hydrodynamic experiments were conducted in a custom-designed water tank. Figure 4 shows the detailed experimental setup. It consists of a stage system (made by Standa Ltd.) for translation control, a minishaker for vibration generation, a sphere to function as a dipole source, and a micro-fabricated 


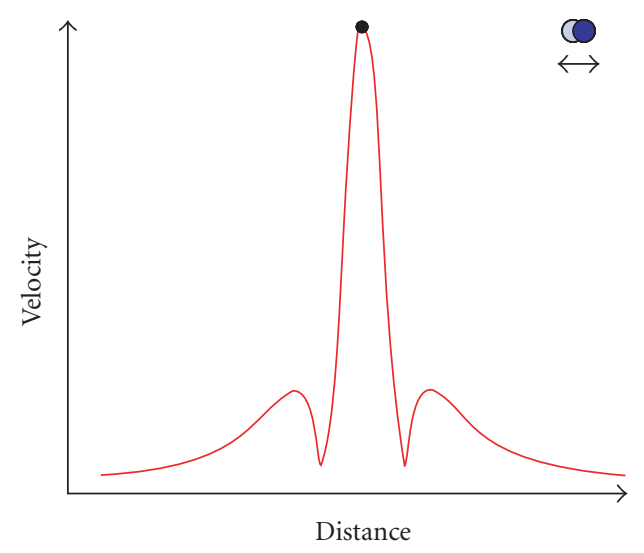

(a)

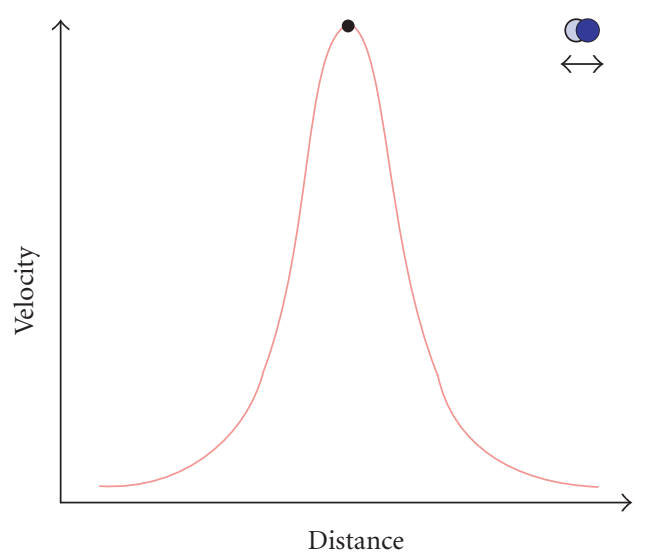

(b)

FIGURE 3: (a) Velocity distribution in response to a dipole (represented as a filled-in circle) as a function of distance away from the dipole ( $x$-axis-along the receiver array) and derived from the analytical model. (b) Velocity distribution of an HWA response to a dipole (represented as a filled-in circle) as a function of distance away from the dipole. In both figures, the oscillating direction of the dipole is shown.

HWA sensor array for sensing and detection. A B\&K minishaker (model 4010) was mounted to the stage system. It can generate sinusoidal vibration along its axis within a frequency range from $2 \mathrm{~Hz}$ to $11000 \mathrm{~Hz}$. A PCB accelerometer (model 352B10) was attached to the rod to measure acceleration of vibration. The sphere vibrated in a direction parallel to the axis of the sensor array, at a fixed frequency of $75 \mathrm{~Hz}$ and displacement amplitude of $0.4 \mathrm{~mm}$.

\section{SIGNAL PROCESSING ALGORITHMS}

We investigated and implemented two approaches to successfully predict the dipole location. These approaches consisted of the template training approach and the modeling approach, both of which operate on empirical data collected using the systems described in Section 4. A minimum mean-squared error (MMSE) algorithm was used in both approaches. As shown in [26], for independent, identically

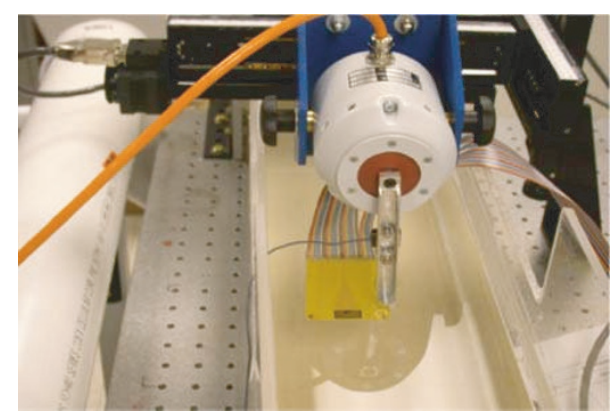

(a)

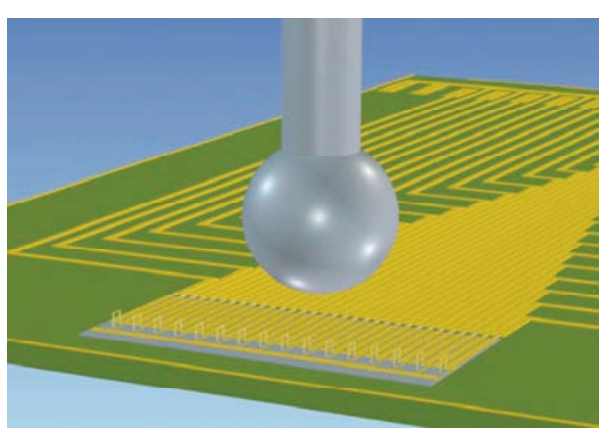

(b)

Figure 4: (a) Overview of the experimental setup. (b) Local details of a dipole source (vibrating sphere) and the HWA sensor array.

distributed Gaussian noise at each sensor (a reasonable assumption for electronic noise), this is also a maximum likelihood estimator (MLE). We describe these approaches and their implementation in detail in the following sections.

The template training approach compared experimental data to a series of templates to make a decision. Two data sets were collected and used. The first data set was called the training data set, or the template set. The second data set was called the experimental data set. Systematic measurements were made with the dipole source traveling step by step in a grid scanning two body lengths of the sensor array along its axis and one body length away from it. Distance away from the array (normal to the array) was designated as the $y$-axis, whereas distance along (parallel to) the array was designated as the $x$-axis. A spatial distribution of the magnitude of flow velocity fluctuation was collected from the lateral line for the dipole source located at each grid point (vertex), with individual grid points $1 \mathrm{~mm}$ apart (Figure 5). Four runs were taken at each specified grid point. For each run, time traces of signal outputs from 16 channels (sensors) were recorded through a computer-controlled data acquisition system via Labview interface, with a sample rate of 2048 samples/s and a total length of 1024 samples for each channel. Later, experimental runs were recorded as the dipole source was mechanically swept along various paths. Three experimental paths are shown-one parallel to the direction of the lateral line (i.e., along $x$-axis), one perpendicular to the lateral line (i.e., along $y$-axis), and one being a zigzagged, inclined path. 


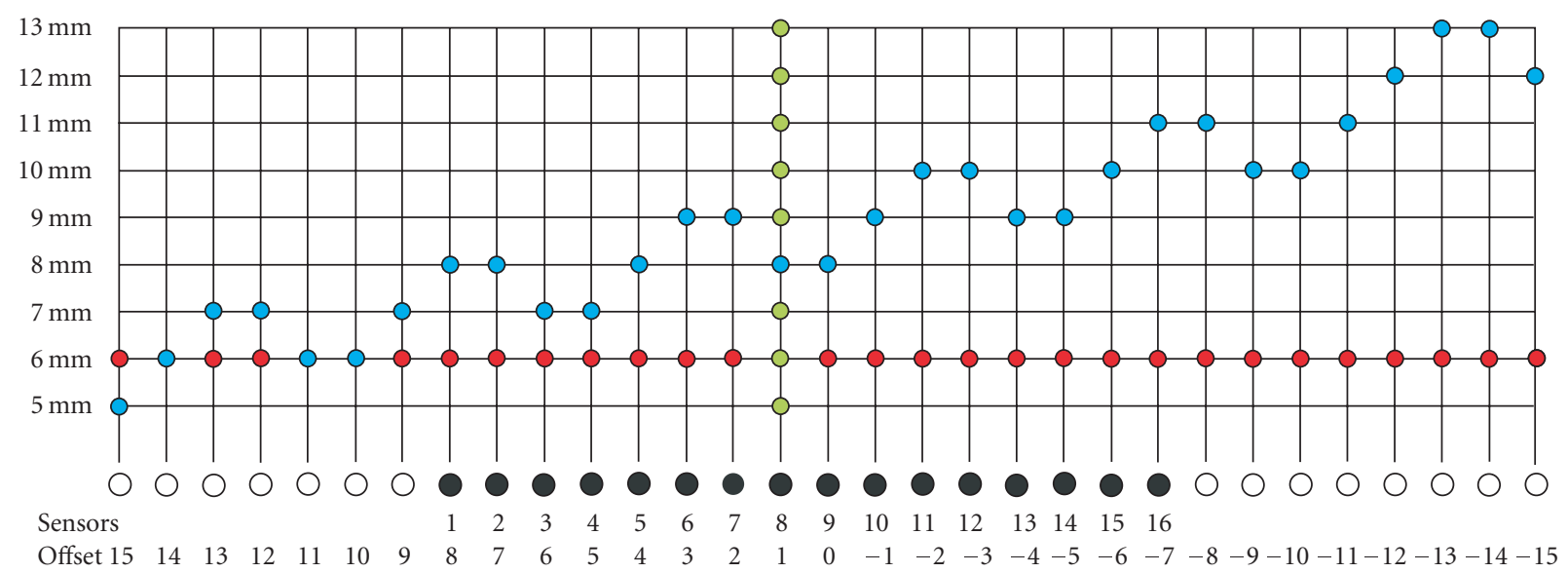

FIGURE 5: The training grid used for recording template and experimental data. The $y$-axis is the distance away from the sensor array (represented by filled-in circles at the bottom). The $x$-axis is the distance along the sensor array. As mentioned, there were three experimental data sets. One experimental data set is along the $x$-axis (horizontal sweep). Another is along the $y$-axis (vertical sweep). The third experimental data set was a zigzagged path.

For each integer position on the $y$-axis (within the relevant scope), a training matrix was created with rows being the horizontal integer positions (31 positions along the array) and with columns being the sensor outputs (16 sensors) averaged over four dipole measurement runs. Effectively, there were 9 positions (5-13 $\mathrm{mm}$ inclusive) vertically, leading to nine training matrices. These were coalesced into a combined three-dimensional matrix, indexed by vertical position first, called the training data set as mentioned above. Each of the experimental data sets consisted of an $m$-by- $n$ matrix, where $m$ is the number of experimental positions and $n$ is the number of sensor outputs.

A minimum mean-squared error (MMSE) estimator was used. We assume that we have a calibrated data training set as well as an experimental data set taken using hardware calibrated in the same manner. For a set of sensor readings corresponding to a particular position $(k)$ in the experimental data set, a search is then performed through the template $x-y$ grid. When the error between the experimental data set under consideration and a particular template is minimal, the $x$ and $y$ coordinates corresponding to that template constitutes the predictive solution. The algorithm is presented in pseudocode in Algorithm 1.

The modeling approach was used in an effort to improve the performance of the training algorithm. A model was empirically developed for the MEMS HWA for this study. Due to the visual form of the data, we speculated that a Gaussian mixture model might work well as an empirical model. Gaussian mixtures of the form of (3) were tried.

$$
f(x)=\sum_{n=1}^{k} a_{n} e^{\left(\left(-\left(x-b_{n}\right) / \sqrt{2} c_{n}\right)^{2}\right)} .
$$

From (3), the variable $k$ is hereto referred to as the order of the fit. The first-order fit suitably approximates the sensor data, while higher-order fits fine-tune the approximation and increase the goodness of fit. Figure 6 shows the approxima-

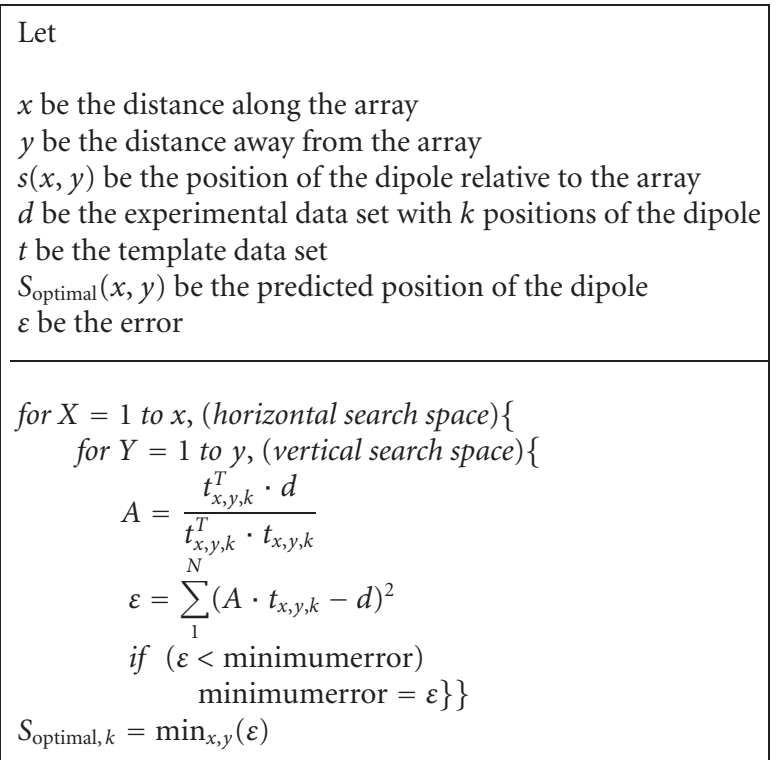

Algorithm 1: (Top) Definition of variables used. (Bottom) MMSE algorithm in pseudocode. $A$ is the correlation factor between the template and data sets for the MMSE algorithm, $\varepsilon$ is the error, while $S$ is the predictive solution.

tion of the data collected by a single MEMS HWA sensor by Gaussian fits of the first and second orders. The first order fit yielded an $\mathrm{R}^{2}$ value of 0.985 while the second (and successive high-order) fit yielded a $0.997 \mathrm{R}^{2}$ value. Polynomial fits were also attempted, but were not used due to the complexity of the high-order curves needed for a good fit. Often, as shown in Figure 6, a ninth-order or higher polynomial curve was needed to achieve a fit with an $\mathrm{R}^{2}$ value of .95 , less than even a first-order Gaussian curve. 


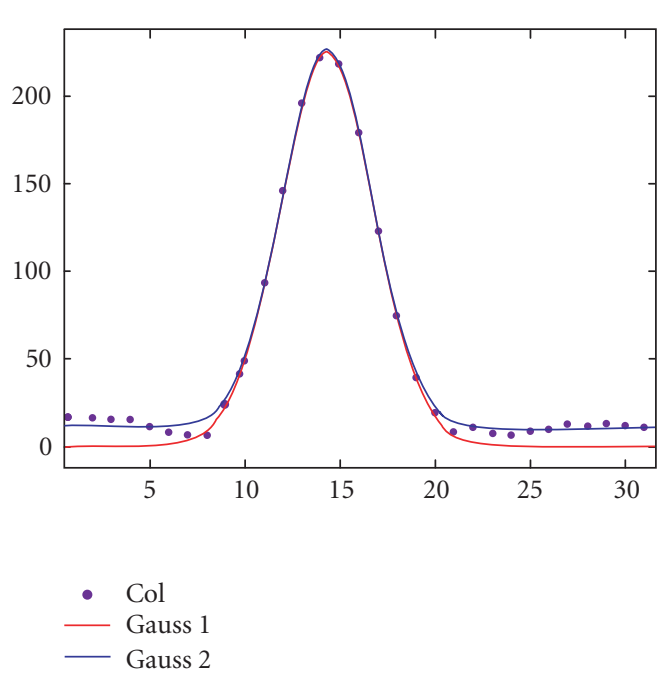

(a)

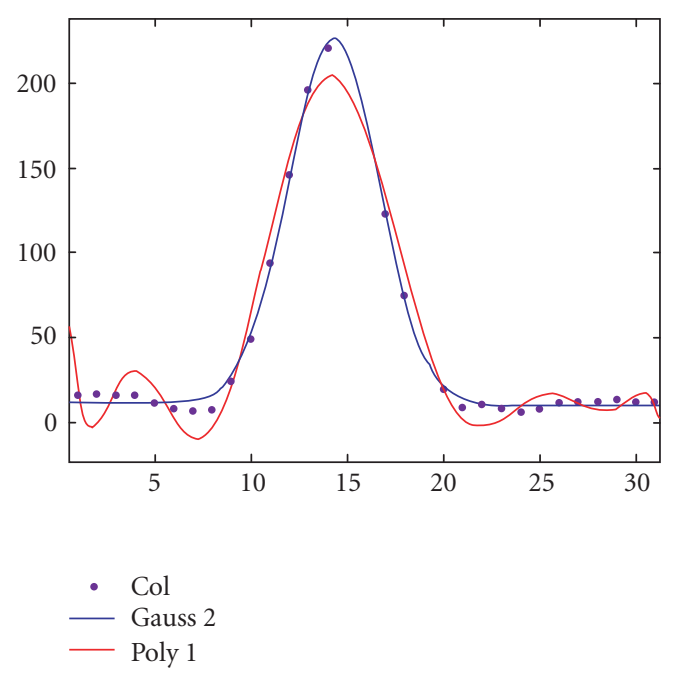

(b)

FIGURE 6: (a) Curve fitting comparison of MEMS sensor data with Gaussian curves. (b) Curve fitting comparison of MEMS sensor data between candidate Gaussian and high-order polynomial curve.

Once the applicable curve was chosen (two-mixture Gaussian), the curve was fit to all 16 columns of sensor training data. Then, the fitted model was used as a template for the MMSE algorithm. The algorithm was designed to predict the position of the dipole to within a millimeter using the Gaussian fit. However, to achieve a greater accuracy (nearest tenth of a millimeter), simple linear interpolation was used between the points of the fit curve. As with training with the sensory data, the MMSE algorithm was used and three experimental runs were conducted as a test of this approach.

\section{RESULTS AND DISCUSSIONS}

The template training approach was used to track the location of the dipole source as it moves through the three representative pathways as described earlier. As shown in Figure 7, the MMSE algorithm accurately predicts the dipole's localization along the array (in the $x$-axis) as well as away from the array ( $y$-axis) in all three experimental cases. For the horizontal sweep, the maximum error in predicting the location of the dipole source is $0.9 \mathrm{~mm}$ in the $x$-axis and $0.5 \mathrm{~mm}$ in the $y$-axis. The average error is $0.1 \mathrm{~mm}$ along either axis. The percentage error of most individual measurements is less than $5 \%$. For the vertical sweep, the maximum error in predicting the location of the dipole source is $0.2 \mathrm{~mm}$ along the $x$-axis and $1.5 \mathrm{~mm}$ in the $y$-axis (vertical axis). The average error is $0.0 \mathrm{~mm}$ in the $x$-axis and $0.4 \mathrm{~mm}$ in the $y$-axis. The percentage error for most of the experimental points is less than $5 \%$ in the $x$-axis and less than $10 \%$ in the $y$-axis. For the zigzag inclined path, the maximum error along the $x$-axis is $0.9 \mathrm{~mm}$ and the maximum error along the $y$-axis is $3.7 \mathrm{~mm}$. The average errors, $0.1 \mathrm{~mm}$ along the $x$-axis and $0.3 \mathrm{~mm}$ along the $y$-axis, are significantly smaller. This is because, statistically, the accuracy for predicting the location of

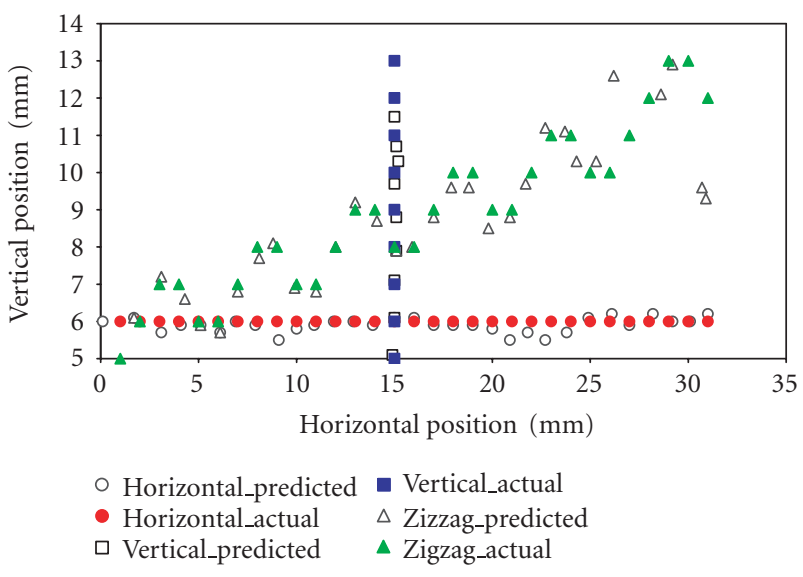

FIGURe 7: Prediction of experimental runs using MMSE algorithm and template training approach.

the dipole decreases as the distance between the dipole and the lateral line increases in both the $x$-axis and $y$-axis. Since a few points on the inclined path are a combination in this regard, the accuracy at the fringe is often limited.

The modeling approach was also used in predicting the location of the dipole source and tracking its movement. Results obtained using this approach are shown in Figure 8. For the horizontal sweep, the maximum error of predicting the dipole source location is $15.6 \mathrm{~mm}$ along the $x$-axis and $7.0 \mathrm{~mm}$ along the $y$-axis. However, these figures are distorted by performance at the fringes. The average error, which holds for most of the points in range of the sensor array, is $0.5 \mathrm{~mm}$ along the $x$-axis and $0.7 \mathrm{~mm}$ along the $y$-axis. For the vertical sweep, the maximum error in predicting the dipole source 


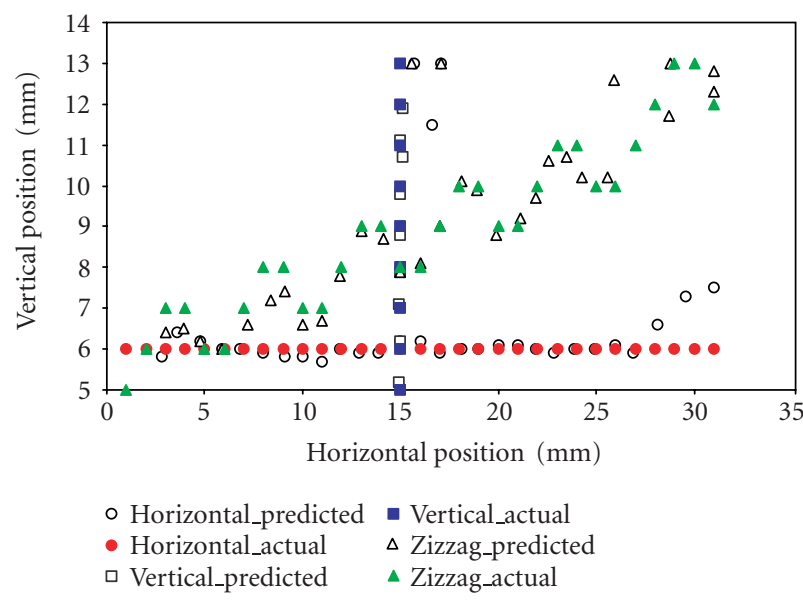

FIgURe 8: Prediction of experimental runs using MMSE algorithm and Gaussian-modeled data.

location is $0.1 \mathrm{~mm}$ along the $x$-axis and $1.1 \mathrm{~mm}$ along the $y$ axis. Once again, outliers distort the performance. The average error is $0.04 \mathrm{~mm}$ along the $x$-axis and $0.3 \mathrm{~mm}$ along the $y$-axis. For the zigzag inclined run, the maximum predictive error along the $x$-axis is $15.1 \mathrm{~mm}$ and $8.0 \mathrm{~mm}$ along the $y$-axis (primarily due to outliers). The average error is $0.9 \mathrm{~mm}$ along the $x$-axis and $0.4 \mathrm{~mm}$ along the $y$-axis. The performance of the modeling approach is similar to the performance of the training approach, but slightly worse due to the inaccuracies of the model. Like the training approach, accuracy at the fringes is low and distorts the overall performance of points within the scope of the array.

We have shown the ability to localize a dipole source using an array of MEMS sensors and bioinspired approaches. The training approach produced accurate results using the MMSE algorithm. Furthermore, the approach can be implemented in a straightforward manner on both static and realtime systems. However, this approach does have its limitations. The computational power and the raw data set (sensory data) need to be significantly large when this approach is applied to complex scenarios. The introduction of variables such as dipole orientation, vibrational frequency and size or a complicated environment involving multiple dipoles would necessitate the use of a much more complex raw data set. Furthermore, the speed and effort of a real-time implementation of the training algorithm would be proportional to the size of the underlying data set.

In contrast, the modeling approach is more flexible. The accuracy of the model can place its performance and limitations anywhere between the formal training to informal heuristics. For our purposes, we used a very accurate model $\left(\mathrm{R}^{2}\right.$ value $\left.>0.99\right)$. At this accuracy, the model closely resembles the underlying data set. Therefore, the model achieves comparable accuracy. The main disadvantage to using a model (Gaussian for the MEMS HWA sensors or analytical model for an ideal dipole) is the difficulty and cost of a system-level implementation. This is due to the fact that the raw data sets must be prefitted to a particular model for the particular array (which requires additional system-level storage) as well as the fact that calibration needs to be done before the approach is initially used.

In a real-world system, such as unmanned underwater vehicle (UUV) guidance or intrusion detection, a hybrid mix of both approaches would be possibly warranted depending on the application goal and engineering constraints. Different applications such as monitoring and targeting for submarines and ships, port and harbor defense, intrusion detection, and hydro-robotics, as well as different environmental conditions might call for a fusion of both approaches.

\section{ACKNOWLEDGMENTS}

The researchers would like to thank their colleagues in the MASS Group as well as collaborators on the DARPA BioSENSE project. This work was funded by the DARPA BioSENSE project through the AFOSR (Program: FA955005-1-0459).

\section{REFERENCES}

[1] S. Coombs, "Nearfield detection of dipole sources by the goldfish (Carassius auratus) and the mottled sculpin (Cottus bairdi)," Journal of Experimental Biology, vol. 190, pp. 109129, 1994.

[2] C. Liu, "Progress in MEMS and micro systems research," in Proceedings of the IMAPS/ACerS 1st International Conference and Exhibition on Ceramic Microsystems Technologies (CICMT '05), Baltimore, Md, USA, April 2005.

[3] C. Liu, Foundations of MEMS, Pearson Education, Upper Saddle River, NJ, USA, 2006.

[4] A. Partridge, J. K. Reynolds, B. W. Chui, et al., "High-performance planar piezoresistive accelerometer," Journal of Microelectromechanical Systems, vol. 9, no. 1, pp. 58-66, 2000.

[5] K. E. Petersen, A. Shartel, and N. F. Raley, "Micromechanical accelerometer integrated with MOS detection circuitry," IEEE Transactions on Electron Devices, vol. 29, no. 1, pp. 2327, 1982.

[6] J. Engel, J. Chen, Z. Fan, and C. Liu, "Polymer micromachined multimodal tactile sensors," Sensors and Actuators A: Physical, vol. 117, no. 1, pp. 50-61, 2005.

[7] J. Engel, J. Chen, and C. Liu, "Development of polyimide flexible tactile sensor skin," Journal of Micromechanics and Microengineering, vol. 13, no. 3, pp. 359-366, 2003.

[8] J. Engel, J. Chen, X. Wang, Z. Fan, C. Liu, and D. L. Jones, "Technology development of integrated multi-modal and flexible tactile skin for robotics applications," in Proceedings of the IEEE/RSJ International Conference on Intelligent Robots and Systems (IROS '03), vol. 3, pp. 2359-2364, Las Vegas, Nev, USA, October 2003.

[9] J. Engel, J. Chen, and C. Liu, "Development of a multi-modal, flexible tactile sensing skin using polymer micromachining," in Proceedings of the 12th International Conference on SolidState Sensors, Actuators and Microsystems, vol. 2, pp. 10271030, Boston, Mass, USA, June 2003.

[10] J. Chen, Z. Fan, J. Zou, J. Engel, and C. Liu, "Two-dimensional micromachined flow sensor array for fluid mechanics studies," Journal of Aerospace Engineering, vol. 16, no. 2, pp. 85-97, 2003. 
[11] C. Liu, J.-B. Huang, Z. Zhu, et al., "A micromachined flow shear-stress sensor based on thermal transfer principles," Journal of Microelectromechanical Systems, vol. 8, no. 1, pp. 90-98, 1999.

[12] J. Chen, J. Engel, N. Chen, S. Pandya, S. Coombs, and C. Liu, "Artificial lateral line and hydrodynamic object tracking," in Proceedings of the 19th IEEE International Conference on Micro Electro Mechanical Systems (MEMS '06), pp. 694-697, Istanbul, Turkey, January 2006.

[13] J. Engel, J. Chen, N. Chen, S. Pandya, and C. Liu, "Development and characterization of an artificial hair cell based on polyurethane elastomer and force sensitive resistors," in Proceedings of the 4th IEEE International Conference on Sensors, Irvine, Calif, USA, October-November 2005.

[14] J. Engel, J. Chen, and C. Liu, "Polymer-based MEMS multimodal sensory array," in Proceedings of the 226th National Meeting of the American Chemical Society (ACS '03), New York, NY, USA, September 2003.

[15] J. Chen, J. Engel, M. Chang, and C. Liu, "3D out-of-plane flow sensor array with integrated circuits," in Proceedings of the 18th European Conference on Solid-State Transducers (Eurosensors XVI), Rome, Italy, September 2004.

[16] J. Chen, J. Engel, N. Chen, and C. Liu, "A monolithic integrated array of out-of-plane hot-wire flow sensors and demonstration of boundary-layer flow imaging," in Proceedings of the 18th IEEE International Conference on Micro Electro Mechanical Systems (MEMS '05), pp. 299-302, Miami Beach, Fla, USA, January-February 2005.

[17] J. Chen, J. Engel, and C. Liu, "Development of polymer-based artificial haircell using surface micromachining and 3D assembly," in Proceedings of the 12th International Conference on Solid-State Sensors, Actuators and Microsystems, vol. 2, pp. 1035-1038, Boston, Mass, USA, June 2003.

[18] J. Chen, Z. Fan, J. Engel, and C. Liu, “Towards modular integrated sensors: the development of artificial haircell sensors using efficient fabrication methods," in Proceedings of the IEEE/RSJ International Conference on Intelligent Robots and Systems (IROS '03), vol. 3, pp. 2341-2346, Las Vegas, Nev, USA, October 2003.

[19] J. Chen and C. Liu, "Development and characterization of surface micromachined, out-of-plane hot-wire anemometer," Journal of Microelectromechanical Systems, vol. 12, no. 6, pp. 979-988, 2003.

[20] J. Chen, J. Zou, and C. Liu, "A surface micromachined, outof-plane anemometer," in Proceedings of the 15th IEEE International Conference on Micro Electro Mechanical Systems (MEMS '02), pp. 332-335, Las Vegas, Nev, USA, January 2002.

[21] S. Coombs, R. R. Fay, and J. Janssen, "Hot-film anemometry for measuring lateral line stimuli," Journal of the Acoustical Society of America, vol. 85, no. 5, pp. 2185-2193, 1989.

[22] P. S. Dubbelday, "Hot-film anemometry measurement of hydroacoustic particle motion," Journal of the Acoustical Society of America, vol. 79, no. 6, pp. 2060-2066, 1986.

[23] Z. Fan, J. Chen, J. Zou, D. Bullen, C. Liu, and F. Delcomyn, "Design and fabrication of artificial lateral line flow sensors," Journal of Micromechanics and Microengineering, vol. 12, no. 5, pp. 655-661, 2002.

[24] S. Coombs, "Smart skins: information processing by lateral line flow sensors," Autonomous Robots, vol. 11, no. 3, pp. 255261, 2001.

[25] S. Coombs, "Dipole 3D user guide," Loyola University, Chicago, Ill, USA, Internal Report and User Guide, 2003.
[26] H. L. Van-Trees, Detection, Estimation, and Modulation Theory, vol. 3, John Wiley \& Sons, New York, NY, USA, 1971.

Saunvit Pandya received his B.S. degree with highest honors in computer engineering from the ECE Department at the Georgia Institute of Technology, where he also was a recipient of the President's Undergraduate Research Scholarship. He is currently an M.S./Ph.D. candidate in the Department of Electrical and Computer Engineering at the University of Illinois at Urbana-Champaign. His interests are in algorithms, ASIC design for DSP and biomimetic MEMS sensors, wireless sensing, sensing and computing architecture, and substrate-to-system integration.

Yingchen Yang received his $\mathrm{Ph} . \mathrm{D}$. degree in mechanical engineering from Lehigh University in May 2005. He is currently a Postdoctoral Researcher in the Micro and Nanotechnology Laboratory at the University of Illinois, involving the development of bioinspired haircell receptive sensors. His research focuses are on flow-structure (sensor) interaction for optimization of sensor design and hydrodynamic trail tracking via application of sensor arrays.

Douglas L. Jones received the B.S.E.E., M.S.E.E., and Ph.D. degrees from Rice University in 1983, 1986, and 1987, respectively. During the 1987-1988 academic year, he was at the University of ErlangenNuremberg in Germany on a Fulbright Postdoctoral Fellowship. Since 1988, he has been with the University of Illinois at Urbana-Champaign, where he is currently a Professor in the Electrical and Computer

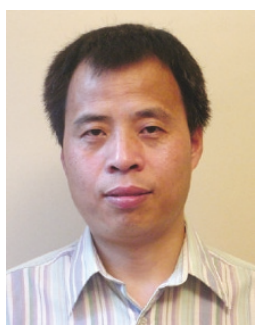
Engineering Department, the Coordinated Science Laboratory, and the Beckman Institute. He was on sabbatical leave at the University of Washington in Spring 1995 and at the University of California at Berkeley in Spring 2002. In the Spring semester of 1999, he served as the Texas Instruments Visiting Professor at Rice University. He is an author of two DSP laboratory textbooks, and was selected as the 2003 Connexions Author of the Year. He is a Fellow of the IEEE. He served on the Board of Governors of the IEEE Signal Processing Society from 2002 to 2004 . His research interests are in digital signal processing and communications, including nonstationary signal analysis, adaptive processing, multisensor data processing, OFDM, and various applications such as advanced hearing aids.

Jonathan Engel received the B.S. degree in general engineering from Harvey Mudd College in 1999 and the M.S. degree in mechanical engineering from the University of Illinois at Urbana-Champaign (UIUC) in 2003. He is working toward the Ph.D. degree at UIUC. From 1999 to 2001, he served as the director of technical sales for MindCruiser Inc. From 2002 to present, he has held a Research Assistantship with the Micro and Nanotechnology Laboratory at UIUC

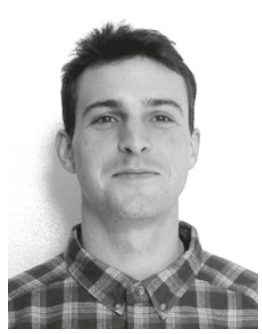


and in 2003 was selected for a Motorola Center for Communications Fellowship through the Coordinated Science Laboratory at UIUC. His research interests include polymer-based and biomimetic MEMS, wireless sensing, as well as fatigue of engineering materials.

Chang Liu received his M.S. and Ph.D. degrees from Caltech in 1991 and 1996, respectively. In January 1997, he became an Assistant Professor with major appointment in the Electrical and Computer Engineering Department and minor appointment in the Mechanical and Industrial Engineering Department. In 2003, he was promoted to Associate Professor with tenure. His research interests cover microsensors,

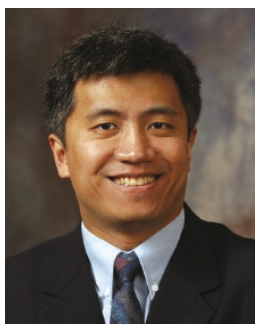

microfluidic lab-on-a-chip systems, and applications of MEMS for nanotechnology. He has 13 years of research experience in the MEMS area and has published 100 technical papers. He received the NSF CAREER award in 1998 and is currently an Associate Editor of the IEEE Sensors Journal. He teaches undergraduate and graduate courses covering the areas of MEMS, solid state electronics, and heat transfer. In 2002, he was elected to the "Inventor Wall of Fame" by the Office of Technology Management of the University of Illinois. 